\title{
Analysis of communication network technology in smart grid
}

\author{
Wang Junsheng ${ }^{1, a}$, OU Qinghai ${ }^{1, b}$, LI Wenjing ${ }^{2, c}$, HE Qingsu ${ }^{3, d}$ \\ ${ }^{1}$ Beijing Huitong Financial Information Technology CO.LTD,Beijing 100053,China; \\ ${ }^{2}$ State Grid Information \& Telecommunication Group. CO. LTD, Beijing 100031, China; \\ ${ }^{3}$ State Grid Information \& Telecommunication Group. CO. LTD, Beijing 100031, China \\ a19158382@qq.com, be-mail: ouqinghai@sgitg.sgcc.com.cn, \\ ce-mail: liwenjing@sgitg.sgcc.com.cn, ${ }^{\mathrm{d} e-m a i l: ~ h e q i n g s u @ s g i t g . s g c c . c o m . c n ~}$
}

Keywords: Smart grid, Communication networking.

\begin{abstract}
Smart grid is built on the integrated communication network, through the sensing technology, measurement technology and control methods for decision-making technology applications. This paper mainly analyzes the communication networking technology in smart grid. This paper focuses on the typical networking technology in the smart grid from three aspects: the sensing extension layer, the access layer and the core layer.
\end{abstract}

\section{Introduction}

Communication network of smart grid are diverse and complex. It covers a variety of technologies, such as wireless broadband access technology, carrier communication technology, optical fiber communication technology, data radio and so on. Communication technology has been applied in different environments. The smart grid communication technology need to be applied to the above a variety of communication technologies. Due to the different communication systems have their own characteristics, thus better integrated of different communication technologies can achieve the requirement of the smart grid communication. The smart grid communication network technology has important implications for the development of smart grid.

\section{The smart grid communication networking technology}

At present, the communication network of distribution system in our country mainly include 
wireless public network, Ethernet passive optical network, optical modems, medium voltage power line carrier, such as industrial Ethernet communication mode ${ }^{[1]}$. Smart grid needs to meet the relevant requirement of power distribution network technology. At the same time, the communication network of the smart grid should also have a video, data, voice and other function.

\section{Perception layer network analysis}

\section{1 wireless sensor networking}

Wireless sensor network (WSN) is a collection of modern theory of sensor, embedded theory, distributed information processing technology and microelectronic mechanical technology development of a new type of network, it consists of random distribution of integrated with sensors, control processors and tiny node communication module composition by means of self-organizing networks $^{[2-3]}$. WSN for IEEE 802.15.4 protocol, the key criteria for the whole of the physical layer and MAC layer has carried on the strict rules, enables the use of the different sensors embedded in the node, or to the environment monitoring object information for real-time monitoring, perception and gathering, and based on the information processing to obtain detailed and accurate information, can be in office when a under various environmental conditions, the vast majority of sites and get a lot of detailed and reliable information.

The characteristics of WSN:

(1) Small size, low cost sensing node is the biggest advantage of WSN

(2)Large number of sensor nodes, is adaptive.

(3)Communication RADIUS, bandwidth is very low.

\subsection{Power Line Carrier networking}

Power line communication technology (Power Line Communication) referred to as PLC technology ${ }^{[4]}$. By using the existing network to transfer voice, data, images and other multimedia business information is the basic principle of it. That is loaded with information of signal transmission load on the power line, at the receiving end through a modem and the signal from the power line by FGC extracted, and transmitted to the computer, communication equipment, Implement the specific business.

Improve power line communication situation and continue to optimize communication coding algorithm and modulation mode, improved data transmission rate and stability, reliability and anti-interference ability.

The advantages of PLC are as follows:

(1)Low cost: It can directly use of the existing power lines as a transmission line, without extra wiring, thereby greatly reducing the network investment, save workload, reduce the cost. 
(2) A wide range: Power line covers a wide range, its wide coverage is unmatched by any other network. So the PLC can easily infiltrate into every family, create a great space for the development of the Internet.

(3)High speed: PLC provides high-speed transmission speed. The mainstream of the PLC communication equipment on the market transmission rate can reach 4.SM-45MBPS. Higher than dial-up and ISDN, even faster than ADSL, can meet different needs of the business of ordinary families.

(4) Always online: Devices of PLC are "plug and play" with no cumbersome dialing process. As long as access to power is equal to the access network.

\section{Access layer networking analysis}

Access layer is a bridge connecting customers to the Internet. Selecting ifferent access technology for the user's experience, network business ability has very big effect. This section will be main access technology.

\subsection{XDSL access networking}

Digital subscriber line (DSL) is the data after a modem connected to the Internet through a telephone line access technology. Because of its make full use of the existing telephone network resources, and its simple connection, small investment, convenient access, it is widely used in the home user's network access.

DSL including high-speed DSL (HDSL), ultra high speed DSL (VDSL), asymmetric DSL (ADSL), ISDN a DSL (IDSL), rate adaptive DSL (high), one-way DSL (UDSL) and the other technology. At present domestic operators mainly provide ADSL access solution for the user, using frequency division multiplexing technology to copper shaft line into the phone, ascending and descending three relatively independent channels. It not only can avoid the mutual interference of the three, but also can provide 3 MBPS uplink speed and 24 MBPS downstream.

Existing DSL standard, such as ITU-T0992, ITU-tCx994, and ANSI T1.413 is already very mature, widely used in business. At the same time, the emergence of ADSL2 and ADSL2 +ADSL has injected new vitality.

XDSL can be used in the industry private network, Internet, ATM network access, etc. Relative to the optical fiber access, however, DSL access scheme of transmission rate is still unsatisfactory, only to take a new modulation and coding technology can further increase the transmission rate of copper wire.

\subsection{Optical fiber access networking}

Optical fiber access technology (FTTx) including optical fiber to the home (FTTH), the optical 
fiber to the curb (FTTC), optical fiber to the building (FTTB), etc. Optical fiber access network can not only meet the needs of users for a variety of business, overcome copper wire cable unable to overcome some of the limiting factors, and low loss, wide frequency band, remove the limitation of the copper wire cable mesh size small. In addition, the optical fiber is not affected by electromagnetic interference, to ensure the quality of signal transmission. Through the optical fiber composite low voltage cables (OPLC) will be optical fiber with low voltage power line laying, smart meters to households, cooperate with the passive optical network technology, carrying electricity information collection, intelligent power two-way interaction, multi-network integration, etc.

As one of the important cable products in the construction of smart grid, OPLC combined with the double function of communication and electric power, which can effectively realize telecommunication network, electric power transmission network, network, Internet, how to merge, reduce the cost of network construction, is the one of the highest cost-effective "multi-network integration" products.

Ethernet passive optical network (EPON) is a new kind of optical fiber access network technology ${ }^{[5-6]}$. It is point to multipoint structure, the passive optical fiber transmission, on Ethernet offers a variety of business. It adopts PON technology in physical layer. Ethernet protocols are used in the link layer, using the topology of the PON implementation of Ethernet access. Thus, it combines the advantages of PON technology and Ethernet technology, such as low cost, high bandwidth, strong scalability, flexible and fast service reorganization, compatibility with existing Ethernet and convenient management, etc.

\subsection{Wireless access networking}

With WiMAX, McWiLL, 3G wireless network as a representative of a series of high-speed wireless communication technology for wireless networks to application in the network access layer. McWiLL. 3G technology are not applicable to electric power information transmission. Because of the public transport information insecurity. WiMAX technology is wireless broadband access technology based on IEEE802. 16 standard. Introduced support for on line-of-sight and mobility, provide higher spectrum efficiency, so as to realize the broadband wireless access ${ }^{[3]}$.

Compared with traditional wired access, wireless access does not need to set up cables, flexible networking and can access the network anywhere, anytime. Wireless channel is also vulnerable to environmental impact of the reliability of data than cable channel is high, the electricity information collection and distribution automation information has a great influence of the grid. Broadcast about the inherent nature of the wireless channel also determines the security of the information can only be done through encryption and private information for power grid maintenance also have potential dangers.

\section{The core networking analysis}

Network core layer is made of optical fiber communication has become a global consensus ${ }^{[7] .}$ 
The optical fiber communication originated from 20 centuries 70 years. Today, it's development speed is very fast, in the telecommunication networks and other aspects have won a wide application. Whether long-distance backbone network or access network, the optical fiber communication became the main means of transport because of its cheap, excellent bandwidth transportion.

\subsection{SDH Networking}

SDH technology not only provided a unified interface rate and light in the world, but also adopted the flexible reusable function, provided rich network management capabilities, but also has the very strong ring network protection function. So, SDH technology now has developed into one of the most widely used fiber optic networking standards.

Data services had already become the most important telecommunications network business. So the traditional circuit switched network to the representatives of the IP network packet network improvement has become an unstoppable trend. Although SDH technology has obvious advantages, but the development of SDH based on it circuit switching mode time slot reuse. So its support for data services is not very good. Independent SDH equipment faced several serious problems:

(1) The time of business deployment is too long

While network management could complete the configuration of the entire network, unfriendly interface led to the initial configuration of the network configuration process is too cumbersome and complicated. Even if the current SDH equipment could be remotely configured and brought great convenience, but after SDH network gradually expanding, traditional manual management very clearly wasted, network configuration for hours, days or even months was not uncommon. If meets the network topology may change at any time, it will lead to the telecom operators will waste a lot of time.

(2) Low resource utilization

In SDH, Ring network protection rearrangement mechanism provides good security for the entire network, but backup circuit always occupy half of the resource. At the expense of the network resources for traditional scheme of network survivability has brought a great waste of resources. More importantly, SDH is originally designed for TDM circuit exchange business. Nowadays the IP data service to become the main business, in order to support IP business, SDH must be combined with other additional technology to host IP technology, which will result in waste every level cost, equipment is also becoming more complicated.

(3) The complexity of network management

Ring network protection rearrangement not only results in SDH resource utilization is too low, but also leads to network management more complex. When setting up the network, Volume is relatively small, less network central number, the topology structure is clear, network management is simple and reliable. But after the business is growing, the network scale expanding, the number of nodes and the ring number also rise accordingly, network management is facing severe challenges. Between various vendors do not have a unified standard of network management unit that caused there is not a unified network management platform for the world to the global first. 
(4) The high cost of maintenance and expansion

SDH Lack of intelligent control technology. Network management requires a lot of manpower, and requires high technical level of staff, so the maintenance cost is high. Network expansion must be based on the ring, and ring network has business bottleneck problems. At the same time, with the number of rings increasing, the expansion of the network becomes more difficult and the expansion costs higher.

(5) Does not offer a good QoS Services

SDH mapped different business to different container without classification, but any container is being treated equally. It wasn't according to its hosting business types, sources, different paying to set priorities. So it couldn't provide a good QoS Supports.

\subsection{ASON Networking}

Automatic switching optical network (ASON) automatically discovered network resources and topology, selected dynamic routing algorithms and distributed signal processing algorithms, to set up an on-demand connection from end to end. Meanwhile, ASON mesh network topology provides reliable protection recovery mechanism, achieves real-time network automatic refactoring when finding the fault, and introduces control plane into optical transmission network, which realizes the on-demand real-time of network resources allocation and finally realizes the comprehensive intelligent optical network.

ASON system is divided into management plane, control plane and data plane. Management plane provides a unified management platform for Network Managers; Control plane control running status of various kinds of network protocols; Data plane is responsible for real-time connection of end-to-end. It directly determines the transmission performance of the equipment.

The interface of ASON plan communication protocol between different function entity equipment, meanwhile as the connection channel. Deliver the appropriate control information to complete connection between switch ports through CCI connection transport plane and control plane. By NMI-A and NMI-T respectively, connection management and control plane and data plane, to implement management face the management of both.

\section{Conclusions}

Communication network is an important part of smart grid. The communication network technology application in the smart grid, can provide people with more secure, reliable, convenient power services. Therefore, the study of communication network technology ensured the development of modern social economy, provided users with reliable safety of electric power communication transmission and speed up the development of the smart grid and economic development has a very important role. 


\section{References}

[1] Shang xiaojun. Development and characteristics of power line carrier communication technology [f]. Power system communications, 2003, 5 (1) : 47-51

[2] J. Goldsmith, S. B. Wicker. Design Challenges for Energy Constrained Wireless Ad Hoc Networks[J]. IEEE Wireless Communications, Aug. 2002: 8-27

[3] M. Gerla and J. Tsai. Multi-cluster, mobile, multimedia radio network[J]. AGM Boltzer Journal of Wireless Networks. 1995, 1(3): 255-265

[4] Khurram,Hussain,Zuberi.Power LineCarrier (PLC) Communication Systems [M]. Department of Microelectronics and Information Technology,2003.

[5] Xu guangnian Ma Xinxiang, Pan kofi etc. Based on the distribution network communication system design and application of EPON technology [fJl power system communication,2008, 29(5): 59-62

[6] Zhou xin,Zhu lan,Wu jiang etc. EPON network research in intelligent electric communication network with si chuan. Post and telecommunications design technology , 2011,(1):53-57.DOI:10.3969/j.issn.1 007-3043.2011.01.013.

[7] Lin mingyu, Xiang wanqin. With the electric communication network technology application research [L]. Journal of chong qing electric power college 2012,17(5):70-73 